\title{
Molecular characterization of the native (non-linked) CD160-HVEM protein complex revealed by initial crystallographic analysis
}

\author{
S. Lenhartová ${ }^{1}$, M. Nemčovič ${ }^{2}$, R. Šebová ${ }^{1}$, M. Benko ${ }^{1}$, D.M. Zajonc ${ }^{3,4}$, I. Nemčovičová1 \\ ${ }^{1}$ Biomedical Research Center, Slovak Academy of Sciences, Bratislava, Slovakia, \\ ${ }^{2}$ Institute of Chemistry, Slovak Academy of Sciences, Bratislava, Slovakia, \\ ${ }^{3}$ Division of Immune Regulation, La Jolla Institute for Immunology, La Jolla, CA, USA, \\ ${ }^{4}$ Cancer Immunology Discovery, Pfizer Inc., San Diego, CA, USA \\ viruivka@savba.sk
}

\begin{abstract}
An increasing number of surface-exposed ligands and receptors acting on immune cells are being considered as a starting point in drug development applications. As they are dedicated to manipulate a wide range of immune responses, accurately predicting their molecular interactions will be necessary for the development of safe and effective therapeutics to enhance immune responses and vaccination. Here, we focused on characterization of human CD160 and HVEM immune receptors whose mutual engagement leads to bidirectional signaling (e.g., T cell inhibition, natural killer cell activation, or mucosal immunity). In particular, our study report on the molecule preparation, characterization and initial crystallographic analysis of CD160-HVEM complex and both HVEM and CD160 in ligand-free form. Despite the importance of the CD160-HVEM immune signaling and its therapeutic relevance, the structural and mechanistic basis underlying CD160-HVEM engagement has some controversial evidence. Some newer studies reported CD160 molecule in monomeric form [1-3], while older reports provided evidence on multimeric form acting on immune cells [4, 5]. In our study, the native non-linked CD160-HVEM complex was co-expressed in the baculovirus-insect host; purified to homogeneity by anion-exchange chromatography to provide missing evidence of trimeric form in solution. The CD160-HVEM crystallized in orthorhombic space group with unit cell parameters that could accommodate one trimeric complex (3:3) in asymmetric unit. Crystals of CD160-HVEM complex, CD160 trimer and HVEM monomer (reported in two space groups) diffracted to a minimum Bragg spacing of 2.8, 3.1 and 1.9/2.1 $\AA$ resolution, respectively.
\end{abstract}

[1] Liu, W.; Garrett, S. C.; Fedorov, E. V.; Ramagopal, U. A.; Garforth, S. J.; Bonanno, J. B.; Almo, S. C. Structure (2019), 27 (8), $1286-1295$ e4.

[2] Kojima, R.; Kajikawa, M.; Shiroishi, M.; Kuroki, K.; Maenaka, K. J Mol Biol (2011), 413 (4), 762-72.

[3] Stiles, K. M.; Whitbeck, J. C.; Lou, H.; Cohen, G. H.; Eisenberg, R. J.; Krummenacher, C. J Virol (2010), 84 (22), 11646-60.

[4] Anumanthan, A.; Bensussan, A.; Boumsell, L.; Christ, A. D.; Blumberg, R. S.; Voss, S. D.; Patel, A. T.; Robertson, M. J.; Nadler, L. M.; Freeman, G. J. J Immunol (1998), 161 (6), 2780-90.

[5] Maiza, H.; Leca, G.; Mansur, I. G.; Schiavon, V.; Boumsell, L.; Bensussan, A. J Exp Med (1993), 178 (3), 1121-6.

Keywords: CD160/BY55; HVEM/TNFRSF14; immune receptor; immunological synapse; receptor-ligand interaction

This research was funded by the contribution of the Slovak Research and Development Agency under the project APVV-14-0839 and continuous project APVV-19-0376; and the contribution of the Scientific Grant Agency of the Slovak Republic under the grant VEGA02/0020/18 and VE-GA-02/0060/21. IN was Marie Curie Fellow financed by programme SASPRO co-funded by European Union and the Slovak Academy of Sciences. The part of the research team was supported by Interreg $V$-A SK-AT cooperation programme by project CAPSID under the contract No. NFP305010V235 co-financed by European Regional Development Fund. 